\title{
CAE Supported Ornamental Flame Product Design-Vortex Flow Guiding with Fuel Lift Mechanism
}

\author{
Wei Long Chen and Fang Lin Chao \\ Department of Industrial Design, Chaoyang University of Technology, Taichung 436, Taiwan, R.O.C.
}

\begin{abstract}
The vortex flame design process was investigated. Combine qualitative and CAE (computer-aided engineering) simulation proposed to fulfill the functional requirement. Guidance channel can produce an ornamental flame with acceptable height. The lift mechanism comprises a supporting member and bearing and allows moving to the predetermined height. It allows a user to add fuel and cleaning base parts safely. Rigid body linkage and flow field simulated enables fine tune of geometric parameters. Functional test and measurement are carried out to confirm the product features. The measured average temperature of the metal chassis is $37.25{ }^{\circ} \mathrm{C}$, and that of glass is $141^{\circ} \mathrm{C}$.
\end{abstract}

Key words: Ornamental flame, flow guiding, convection, lift mechanism, computer aided engineering.

\section{Introduction}

Over time, the purpose of fireplaces has changed from one of necessity to one of visual interests. Early fireplace or flame devices were used for warmth as a gathering place within the home. In recent years, the design is not only functional but also emotional which allows families to communicate with each other in a relaxed context [1]. Stylish flame affects the atmosphere of the room. Usually, ornamental flash with viable long flame path adds aesthetically pleasing to people.

Decorative flames have gradually been welcomed by families. We must pay attention to the safety issues. Flame devices can be fueled by natural gas, biomass, and propane fuel sources. When a traditional woodblock burns, smoke can cause disease because it associated with lower respiratory tract infections [2]. Ventless fireplaces are usually fueled by either gel, ethanol, or natural gas. Biofuel produced through contemporary biological processes is popular now. Gel fuel and bio-ethanol dedicated to portable fire pits [3]. The difference between gel fuel and liquid

Corresponding author: Fang Lin Chao, Ph.D., associate professor, research field: eco-design. bioethanol is safety. The gel is not natural to diffuse and prevent large-area combustion after pouring. Airflow guidance structure is the central element to produce the ornamental flame with acceptable height. Vortex flame with stable flow pattern is a prefer design target [3].

Sustainable design is assessed usually based on functional, economic, and ecological criteria to achieve sustainability. With sustainable principle in mind, designers get chance to reduce the environmental impact in an early stage. The checklist [4] supports for the analysis of a product's impact on the environment without missing significant features. The main strategies are (1) selection of low-impact materials, (2) reduction of material usage, and (3) modification of production techniques, and (4) reduction of impact during use [5].

\section{Design Process}

The design process has to meet functional requirements:

- flame guiding structure to produce acceptable flame height,

- the lift mechanism allows users to ignite the fuel and to remove the fuel container, 
- low center of gravity to ensure the stability,

- the safety concern to prevent accidental operation.

The multi-disciplinary design requires a process to reduce contradictory within the design team. CAE (computer-aided engineering) is an essential step to identify product functional status. Qualitative guidelines are crucial to finding design solutions in the early stage.

\subsection{CAE in Sustainable Design}

Detailed calculation steps by CAE can support engineer to get right parameters. The abilities of prediction and insight of problem make CAE simulation important in sustainable design. The burning and heat transfer mechanisms caused the high temperature of body and parts. CFD (computational fluid dynamics) techniques are the foundation for predicting airflow. A finite volume method is a conventional approach for large problems, and source term dominated flows. The structure designs allow user to perform the task quickly without potential safety issues.

\subsection{Qualitative Guidelines}

General design guidelines enable the designer to understand trend and significant factors. To maintain production cost and reduce raw material used, the size and shape of the airflow guiding channel need preserve ventilation path with minimum turbulence. High surface temperature causes user risk. From non-quantitative design guidelines, we can not only transfer the experiences but also prevent pitfall that may happen. Those cases are collected in different domains such as computer chassis, avionic facilities. The examples of the flow guidelines are as follows $[6,7]$ :

- Airflow reversal may happen if inlet velocity is too high.

- Do not place the inlet of a blower directly downstream of the exhaust of another blower.

- Do not obstruct coolant flow, guide flow using splitters and turning vanes.
- Use gradual turns, enlargements, and contractions in flow passages.

- Install cooling fins vertically. Do not place parts directly above high dissipating elements.

The fluid characteristic of different domain remains the same. For example, airflow guidance structures: Fig. 1a change channel diameter and flow direction; Fig. $1 \mathrm{~b}$ turning loss coefficients for a branch, and Fig. 1c flow reversal due to low static pressure are a common phenomenon. The effect of "Airflow reversal may happen, if inlet velocity is high" can be found in the real-world situation. As the velocity increased, the static pressure will decrease. High air velocity can result in such a low static pressure at the inlet [6]. When a flow reversal happens, the hot air will then be drawn in from the exit plenum. In Fig. 1d, a tapper inlet channel is used so that the cross-section area at any point is higher than that of downstream.

\subsection{Lift Mechanism}

Product structures must allow the user to add fuel and cleaning base efficiently and safely. Mechanical CAE allows three-dimensional demonstration of moving parts operating in conjunction with one another. Multibody dynamics get right parameters and prevent ill mechanism.

In Ref. [8], CAE was utilized to motivate student's creativity in product design. Authors found the importance of pre-defined evaluation criteria for benchmarking a high number of potential mechanisms. Students put a sketch of their solution in a common folder and shared directory concept with group members. In Ref. [9], a lift system incorporates a transverse bar which carries a patient lift system for movement between two laterally extending bars. The transverse bar is mounted at the same vertical height as the laterally extending bars and carries a motor for lifting. In our design, evaluation criteria were set by the available benchmark. The CAE is then utilized to determine suitable parameters that fit pre-defined compact space. 


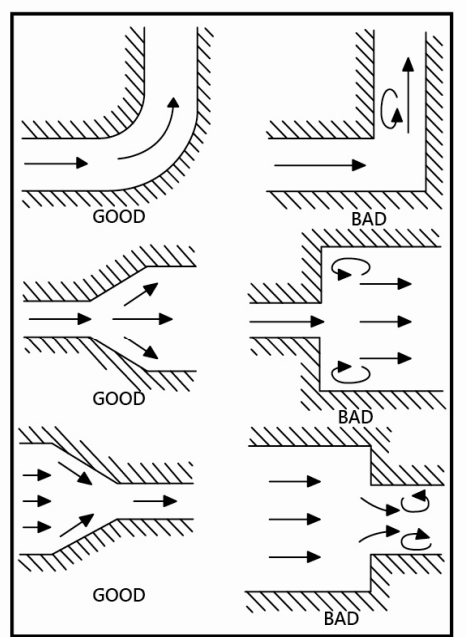

(a)

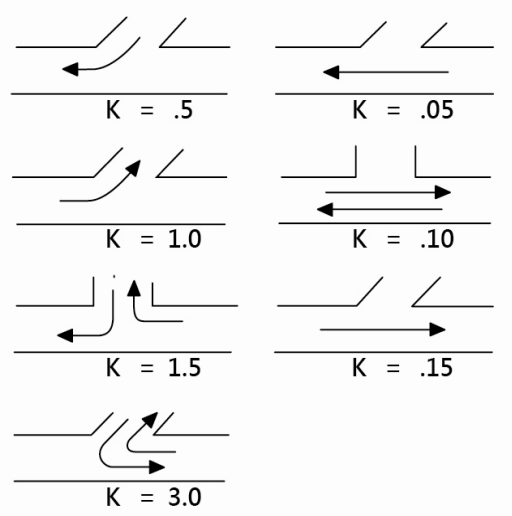

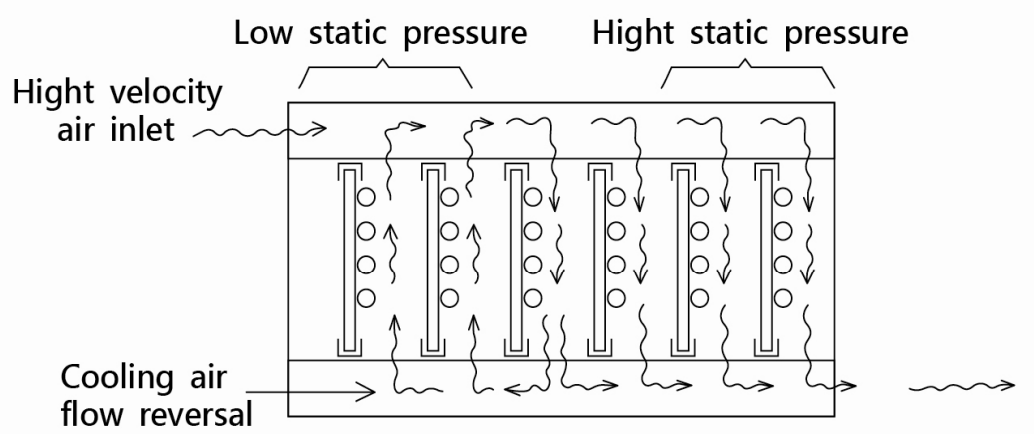

(c)

(b)

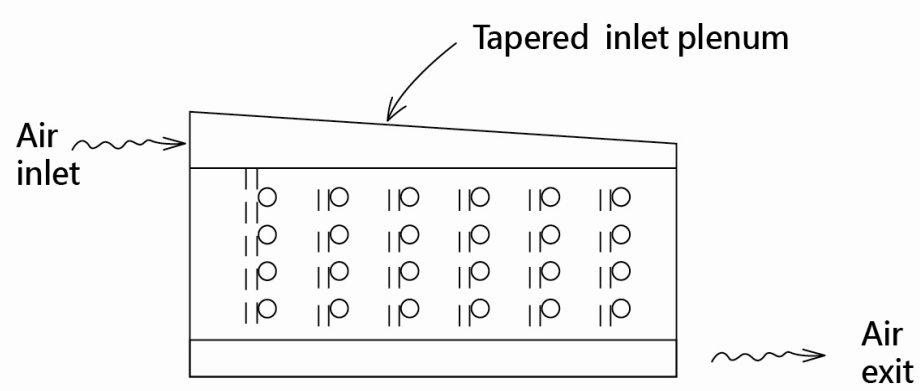

(d) exit

Fig. 1 (a) Use gradual turns, enlargements, and contractions inflow passages; (b) Turning loss coefficients for fluids [6]; (c) Air flow reversal may happen if inlet velocity is high; (d) Improvement in the plenum design by tapper channel [7].

\section{The Case Study of Flame Design}

For enhancing usability, airflow guidance structure cannot interfere with the fuel intake areas. The design goals are within space constraint.

- Changing guidance channel that produces an ornamental flame with acceptable flame height;

- Choosing mechanism that can move vertically without skew to ensure fuel will not spill out.

\subsection{Design Procedure}

Low oxygen consumption biofuel can produce an ornamental flame. First, the digital model was built using CATIA (computer-aided three-dimensional interactive application) solid modeling package. Depending on the type of simulation (flow or mechanism), digital model was discretized into the mesh for setting up the system equation with boundary conditions. Static solutions of field distribution or time depended on the motion obtained by solving the linear matrix equations.

The prototype model was built with the actual size and material so that engineer can measure corresponding physical parameters. The measured data are utilized to adjust the simulation parameters iteratively till results meet each other. While the simulation parameters confirmed, digital model confidently presents a real system which reduces project developing cycle time. 


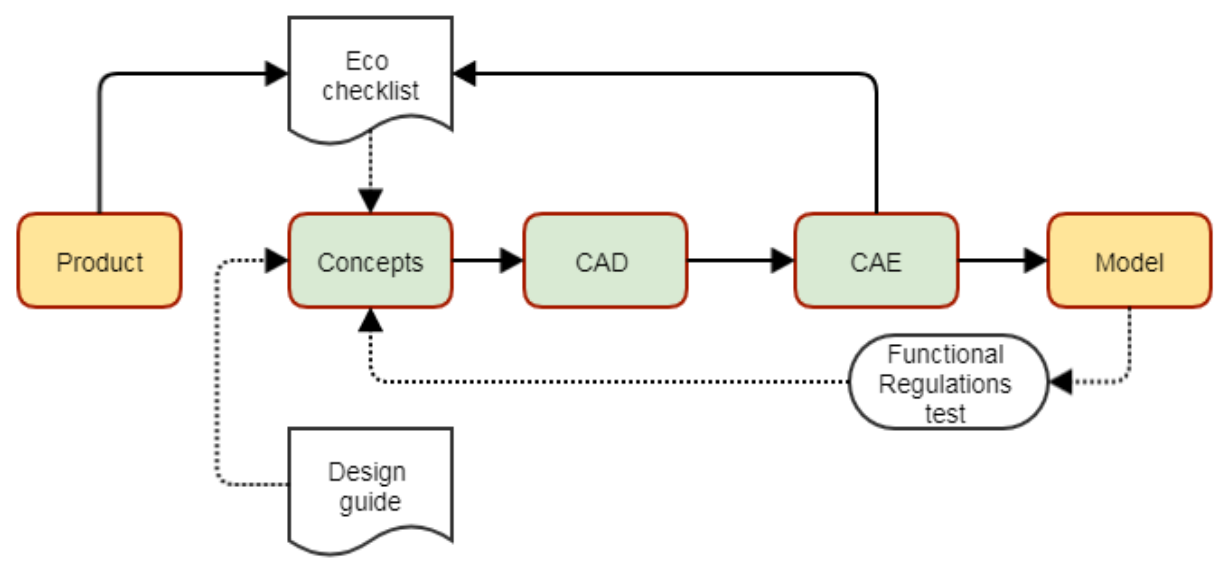

Fig. 2 Design process.

As shown in Fig. 2, the design guideline helps prevent mistake arrangements. $\mathrm{CAD} / \mathrm{CAE}$ is used to verify the idea. The flow field or mechanism simulation enables fine-tuning before a series of measurements. After CAE the Eco checklist was reviewed to confirm the sustainable design.

\subsection{Flow Simulation Results}

When the flame is small, when the length of the flame mask is not long enough, a significant rotating flame effect cannot be formed. To produce a vortex ornamented flame, particular flame guided channel is needed. Flow simulation verified the parameters of the guided channels.

The complicated phenomena of flame make CAE simulation harder. Flow channel analysis was performed using non-premixed combustion mode. With the alcohol fuel combustion equation to make sure the amount of oxygen supply is sufficient.

$$
\mathrm{C}_{2} \mathrm{H}_{5} \mathrm{OH}+3 \mathrm{O}_{2} \rightarrow 2 \mathrm{CO}_{2}+3 \mathrm{H}_{2} \mathrm{O}
$$

In non-premixed combustion, fuel and oxidizer enter the reaction zone in distinct streams. Under certain assumptions, the thermochemistry can be reduced to a single parameter: the mixture fraction. The mixture fraction is the mass fraction that originated from the fuel stream. The profiles of scalar properties provide an alternative thermochemical relationship to that of chemical equilibrium [10].
Velocity vector of $\mathrm{XZ}$ section (Fig. 3) indicates the oxygen supply is sufficient. The upwardly convection is on the outer edge of the cylinder. The overall flow line velocity diagram is helically raised. Table 1 suggests parameters based on simulation and measurement.

Through the adjustment of the size of the air inlet and the length of the runner, the angle of the splitter vane is used to guide the lower air and rotate it to accelerate (Fig. 4). At the outset of the flow channel, the cold air outside is along the inner wall of the glass, and an upward airflow is formed due to the centrifugal force. The long glass tubes encourage the chimney effect, and the hot air continues to rise [11].

\subsection{Mechanism Simulation Results}

A structure lift fuel container to a predetermined height, it includes a base, a cover, and a lift mechanism. The base consists of a chamber with an opening and fuel the container (Fig. 5). The cover is movably engaged with the support and selectively closes the opening. The lift mechanism includes a supporting member adapted for sliding. This supporting member being moved down from the predetermined height to the different height allows a user to ignite the fuel safely and to facilitate the removal of the fuel container.

By lifting the fuel tank onto the blade ring, it makes the product more convenient to use. As the use of 
Table 1 Analysis results of exhaust port velocity.

\begin{tabular}{lll}
\hline Quantization value & Entry value & Outlet value \\
\hline Amount of airflow & $3.1995(\mathrm{~g} / \mathrm{sec})$ & $3.278(\mathrm{~g} / \mathrm{sec})$ \\
Oxygen content ratio & 0.232 & 0.1948 \\
Average speed & $0.120(\mathrm{~m} / \mathrm{sec})$ & $1.273(\mathrm{~m} / \mathrm{sec})$ \\
\hline
\end{tabular}
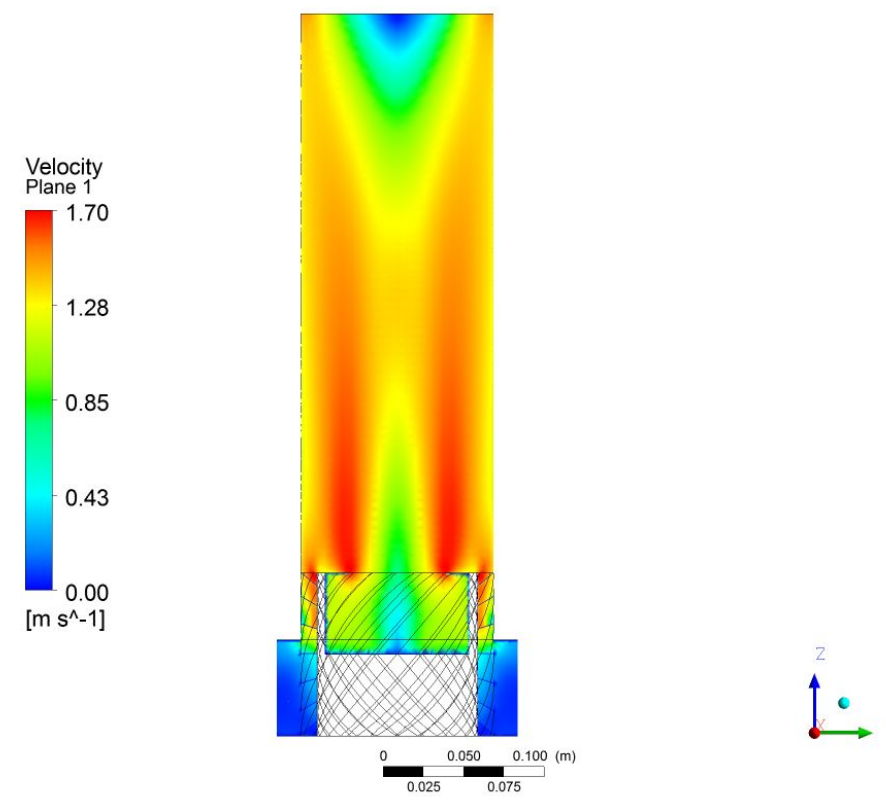

Fig. 3 The velocity of $\mathrm{XZ}$ section, aluminium castings, and iron plate metal, metal contact temperature is not higher than 60 ${ }^{\circ} \mathrm{C}$ (transparent parts: heat-resistant glass).

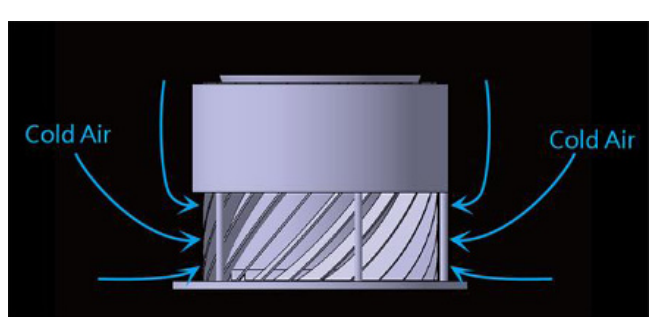

Fig. 4 The adjustment of the size and skew angle of the air channel to guide the cold air and rotate it to accelerate upwards.

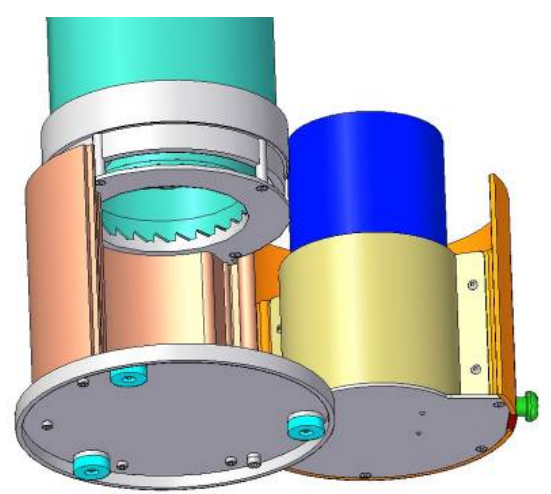

Fig. 5 Perspective view of the fuel container and lift mechanism where the cylinder (inside) is the fuel tank. lifter is very often, different travel range was analyzed (Fig. 6). Fuel tank is placed under the plane pressure of $50 \mathrm{~N}$ (five times of the weight). The material of the fixed point located at the bottom of the four screw holes is aluminum, and stress and deformation were analyzed. The stress is mainly concentrated on the pin connecting area of the hitch and the push rod at $50 \%$ upward travel. The nail in the middle has a small amount of stress concentration, the maximum stress is $43.335 \mathrm{~N}$, and the safety factor is 6.461 . Note that the container keeps in horizontal posture for maintaining ethanol-based fuel staying.

\subsection{Detail Design}

Fig. 7 explodes perspective view of product parts. The cover (\#2) is movably engaged with the base and selectively closing the opening. The cover includes a peripheral wall including a receptacle (\#25) extended therein. The first fixing edge is pivotally engaged with 

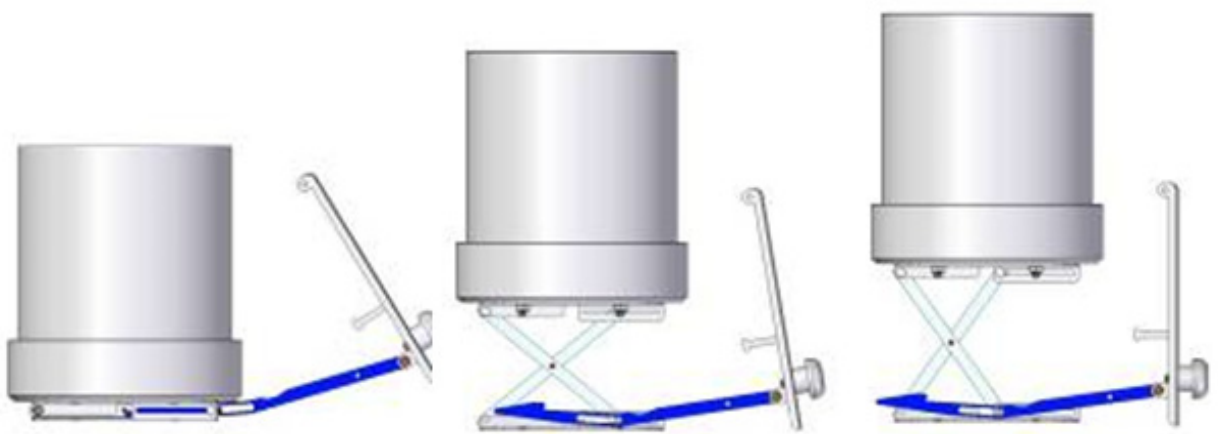

Fig. 6 A cross-sectional view of lift mechanism during moving.

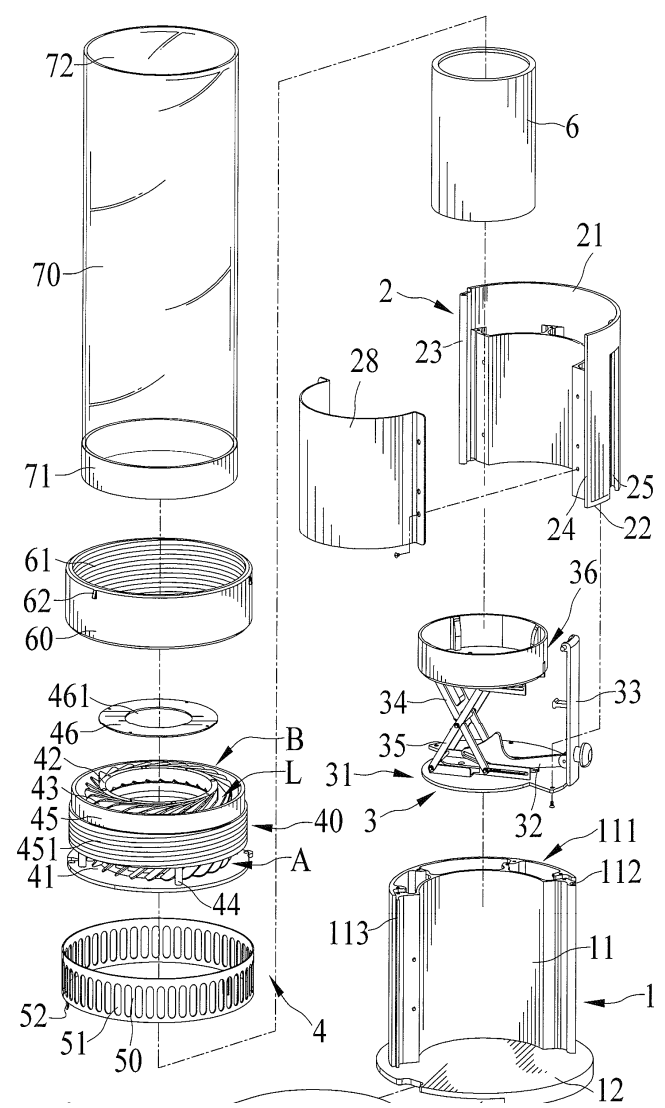

Fig. 7 Parts of the flame device (exploded perspective view) [11], where (\#42) is flow guidance rib, and channel, (L) indicates skew angle, $(\# 40)$ is a module, $(\# 12)$ is base, and (\#34, \#35) are the linkage bar.

the base, with the first fixing edge pivotally engaged with the first engaging edge (\#112) of the lateral wall (\#11) of the bottom.

Product size is: width: $250 \mathrm{~mm}$, height: $460 \mathrm{~mm}$. Glass translucent cover part is $80 \mathrm{~mm}$ or more in diameter and $250 \mathrm{~mm}$ in height. The eight helical guide vanes can produce excellent stability and an attractive viewing vortex flame. The design of the flow path in the air intake area enables the external cold air to enter the guiding structure; therefore, it can maintain the relatively low temperature of the product parts when they are used for a long time.

\subsection{Estimation of the Center of Gravity}

To ensure the stability of the flame device, we need to keep the center of gravity in a lower position. The digital model built within CATIA was verified with appropriate geometric and material properties. A basement made of cast iron was added in outside of the lateral wall (\#11) to maintain a low center of gravity. The calculated center of gravity is indicated in Fig. 8.

When gel fuel is ignited and burned, an oxide layer forms on the surface of the jelly fuel, which

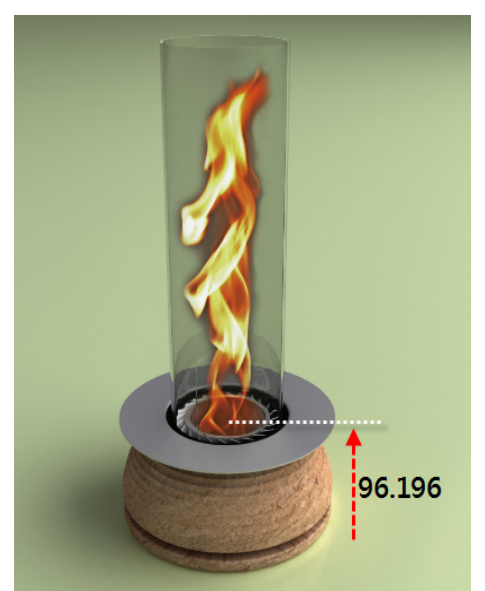

Fig. 8 Calculated center of gravity of flame device: $x=0$ $\mathrm{mm}, y=0 \mathrm{~mm}, z=96.196 \mathrm{~mm}$. 


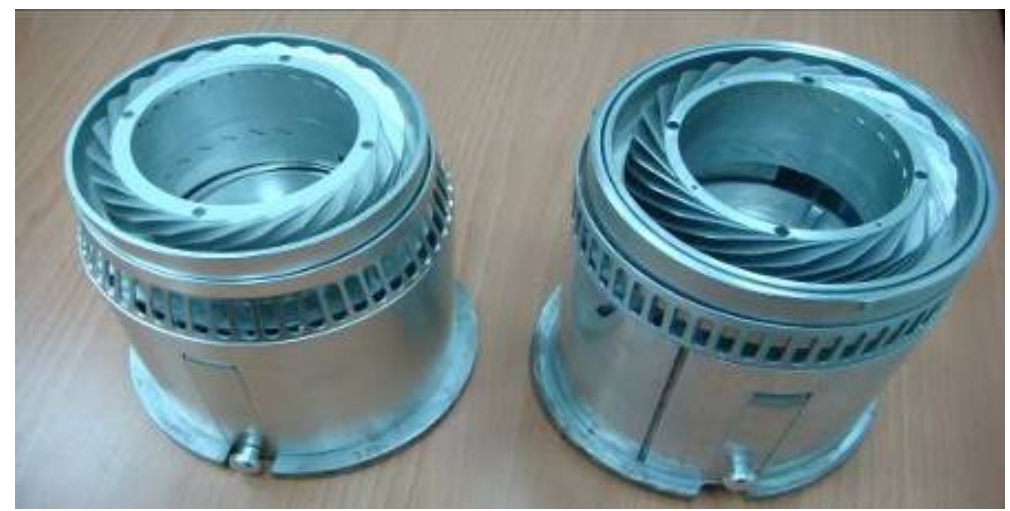

Fig. 9 Prototyping model with fuel tank lifting mechanism shows the detail of the guide vane.
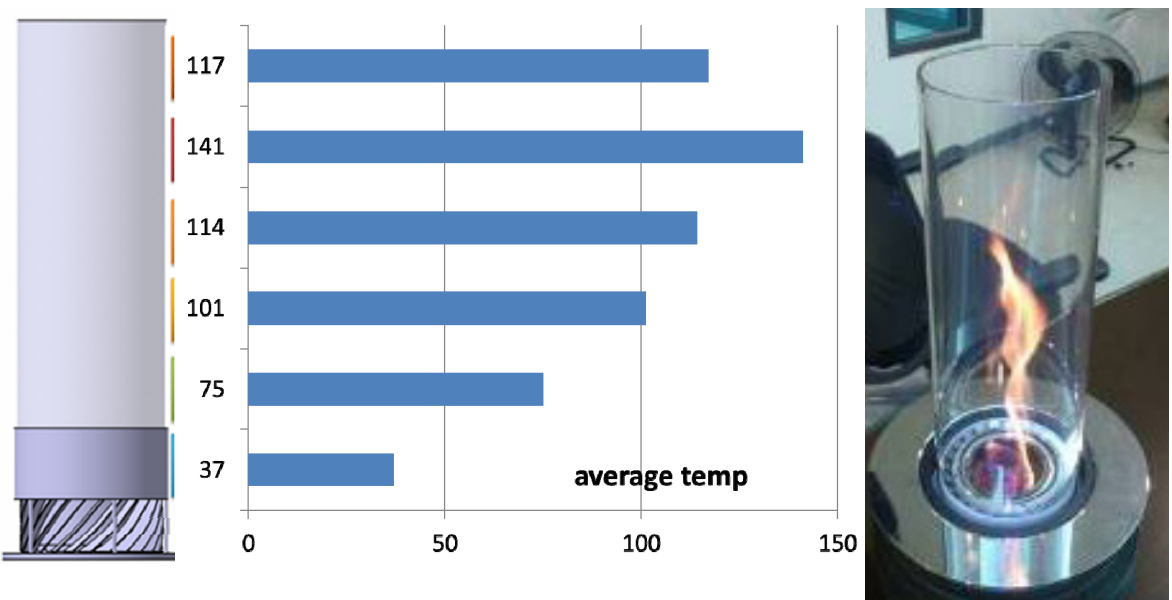

Fig. 10 Measurement of surface temperature uses the prototyping model.

The average temperature of the metal chassis is $37.25^{\circ} \mathrm{C}$ which is touchable with a finger; and that of the glass: the highest part (upper section) is $141^{\circ} \mathrm{C}$ and that of the central section is $114^{\circ} \mathrm{C}$.

slows down the burning rate. When it dumped, the flame becomes smaller due to lack of oxygen, and it is more likely to extinguish. When placed in a semi-open space, the problem of incomplete combustion of carbon monoxide is not yet a problem.

\section{Prototyping Measurement}

Prototyping models (Fig. 9) are used in the test to find better parameters. The flame height of redesign module is $24 \mathrm{~cm}$. The low diversion angle (below 40 degrees) caused outlet oxygen ratio to slightly increase (complete flammability). The main bodies of the ring and spiral material are steel. According to the regulations, we surveyed the CSA (Canadian Standards Association) specification for the stove. There is specification about the glass part of the open fire, but there is no high-temperature limit.
Manufacture need attach warning mark on the glass. The thermal measurement showed the average temperature of the chassis is $37.25^{\circ} \mathrm{C}$ (Fig. 10) which is touchable with a finger; the glass temperature is 114 ${ }^{\circ} \mathrm{C}$ which is also lower than existing products. It revealed the effectiveness of the design process and CAE simulation.

\section{Conclusions}

Through the adjustment of the shape of the air inlet and the length of the runner and the angle of the vane, ornamental vortex flame is achieved. Unique flash guided channel provides the cooling air intake and rotates spirally. At the outset of the guide channel, the cold air goes along the inner wall of the glass, and an upward airflow is formed. The long glass tubes encourage the chimney effect and keep the hot air 
continue to rise. The design process which combines qualitative, CAE and checklist is valuable for the multi-disciplinary project. The study shows a simplicity structure which maintains low surface temperature. CAE helps understand natural convection and the parameters of the four-bar linkage. Through the prototype model tested, we modify and verify the simulation parameters with physical data. With multi-disciplinary principle in mind, designers have a chance to provide safety features in the early design stage.

\section{References}

[1] https://en.wikipedia.org/wiki/Fireplace.

[2] "Derek Kehler and Helena Curic Identified as Young Couple Killed in Kurrajong Cabin.” The Sydney Morning Herald.

[3] REN21. 2011. Renewables 2011: Global Status Report, 13-4.

[4] Ramani, K., Ramanujan, D., Bernstein, W., and Zhao, F. 2010. "Integrated Sustainable Life Cycle Design: A
Review.” ASME J. Mech Design, 132.

[5] Honke, K. S., and Kaebernick, H. 2005. "An Integrated Framework for Implementing Sustainable Product Development." In Proceedings of the 2005 Environmentally Conscious Design and Inverse Manufacturing, Eco Design Fourth International Symposium, 684-91.

[6] Morrison, G. N. 1983. Thermal Guide for Reliability Engineer. Hughes.

[7] Steinberg, D. S. 1980. Cooling Techniques for Electronic Equipment. John Wiley \& Sons.

[8] Fauroux, J. C., Bouzgarrou, B. C., and Gogu, G. 2004. "Innovative Mechanism Design with CAE Software." In DS 33: Proceedings of the 7th International Conference on Engineering and Product Design Education, Delft, the Netherlands, 2-3 September 2004.

[9] Capaldi, G., and Sinreich, M. G. 1998. U.S. Patent No. 5,809,591. Washington, DC: U.S. Patent and Trademark Office.

[10] Liew, S. K., Bray, K. N., and Moss, J. B. 1981. "A Flamelet Model of Turbulent Non-premixed Combustion." Combustion Science and Technology 27 (1-2): 69-73.

[11] Chen, W. L. 2013. US Patent Pub. No. 2013/0011800 A1. 